\title{
Chiral observables and topology in hot QCD with two families of quarks
}

\author{
Florian Burger, ${ }^{1}$ Ernst-Michael Ilgenfritz, ${ }^{2}$ Maria Paola Lombardo, ${ }^{3, *}$ and Anton Trunin ${ }^{4}$ \\ (tmfT Collaboration) \\ ${ }^{1}$ Humboldt-Universität zu Berlin, Institut für Physik, 12489 Berlin, Germany \\ ${ }^{2}$ Joint Institute for Nuclear Research, BLTP, 141980 Dubna, Russia \\ ${ }^{3}$ Laboratori Nazionali di Frascati, INFN, 100044 Frascati, Roma, Italy \\ ${ }^{4}$ Samara National Research University, 443086 Samara, Russia
}

(Received 25 May 2018; published 5 November 2018)

\begin{abstract}
We present results on QCD with four dynamical flavors in the temperature range $150 \mathrm{MeV} \lesssim$ $T \lesssim 500 \mathrm{MeV}$. We have performed lattice simulations with Wilson fermions at maximal twist and measured Polyakov loop, chiral condensate and disconnected susceptibility, on lattices with spacings as fine as $0.065 \mathrm{fm}$. For most observables spacing effects are below statistical errors, which enables us to identify lattice results with continuum estimates. Our estimate of the pseudocritical temperature compares favorably with continuum results from staggered and domain wall fermions, confirming that a dynamical charm does not contribute in the transition region. From the high-temperature behavior of the disconnected chiral susceptibility we infer the topological susceptibility, which encodes relevant properties of the QCD axion, a plausible dark matter candidate. The topological susceptibility thus measured exhibits a power-law decay for $T / T_{c} \gtrsim 2$, with an exponent close to the one predicted by the dilute instanton gas approximation (DIGA). Close to $T_{c}$ the temperature-dependent effective exponent seems to approach the DIGA result from above, a behavior which would support recent analytic calculations based on an instanton-dyon model. These results constrain the mass of a hypothetical QCD post-inflationary axion, once an assumption concerning the relative contribution of axions to dark matter is made.
\end{abstract}

DOI: 10.1103/PhysRevD.98.094501

\section{INTRODUCTION}

The properties of strong interactions at high temperatures are under active scrutiny both theoretically and experimentally. From an experimental viewpoint, collisions of ultrarelativistic ions at the LHC create matter whose temperature has been estimated to reach $500 \mathrm{MeV}$ or more. Theoretically, the behavior of the theory at high temperature offers important insights into the mechanisms of chiral symmetry, confinement and the degrees of freedom (d.o.f.) active in the different phases. For instance, both experiments and lattice results indicate that the system remains very strongly coupled above the critical temperature $T_{c}$, and that the perturbative regime, and eventually a

\footnotetext{
*Address after October 1: INFN sezione di Firenze, Via G. Sansone 1; I-59100 Sesto F.no, Italy

Published by the American Physical Society under the terms of the Creative Commons Attribution 4.0 International license. Further distribution of this work must maintain attribution to the author(s) and the published article's title, journal citation, and DOI. Funded by SCOAP ${ }^{3}$.
}

free-field behavior, will only set in at much higher temperatures; see e.g., Ref. [1].

As a consequence of this, fundamental d.o.f. manifest their influence in a sequential way when temperature increases: particles of the light spectrum dissolve first, and heavy bound states dissolve later. Among the quarks themselves, the light ones, which receive most of their masses at the quark-gluon transition, have a significant influence on the transition itself: the dependence of the value of the pseudocritical temperature(s) as well as of the nature of the transition on the masses of light current quark masses, as well as the role of the strange quark mass around $T_{c}$ have been studied at great length, and the current status of our understanding of the sensitivity of the phase diagram to the light d.o.f. is for instance summarized in the so-called Columbia plot [2-4]. Closely related with the issue of the nature of the phase transition is the (effective) restoration of the $U_{A}(1)$ symmetry $[5,6]$.

By contrast, we know that the charm quark mass does not carry any influence on the transition: if we were to draw a multidimensional Columbia plot including the charm mass $m_{c}$ the plot would just look the same for values of the 
charm mass ranging from infinity down to the experimental value. This can be anticipated just by considering that the charm does not acquire its mass across the chiral phase transition, and our numerical results will confirm this. However, as the temperature increases the role of the charm becomes more important, and hard thermal loop calculations predict a temperature threshold for sizable charm effects at about $300 \mathrm{MeV}$ [7].

This suggests that precise calculations of QCD thermodynamics in the LHC working region, where temperatures as high as $500 \mathrm{MeV}$ may be reached, require a dynamical charm. However, in comparison with studies with $2+1$ flavors, lattice results with two dynamical families are much less developed: lattice discretization effects are more severe due to the large charm mass. Only the MILC Collaboration [8] and the Wuppertal-Budapest Collaboration [9] have published results for the equation of state with $N_{f}=$ $2+1+1$ flavors based on the staggered action. Our own study of the equation of state is underway and only preliminary and partial results have appeared [10], which will not be presented here.

In this paper we study the chiral and topological properties of QCD for temperatures ranging from about $150 \mathrm{MeV}$ to about $500 \mathrm{MeV}$, well above the deconfinement transition, and close to the charm threshold. We use four flavors of maximally twisted mass Wilson fermions in the isospin limit, i.e., with degenerate up and down quark masses, and physical strange and charm masses, relying on the zerotemperature results of the ETM Collaboration (ETMC) for scale setting and tuning of the algorithm to simulate at maximal twist [11].

The purpose of this study is twofold. First, we present the first results around the pseudocritical temperature obtained with twisted mass Wilson fermions. We successfully crosscheck our results with earlier ones obtained with $N_{f}=$ $2+1$ staggered and domain wall fermions [12-14], thus confirming that a dynamical charm has no influence around the transition. Second, we use the results for the disconnected chiral susceptibility to calculate the topological susceptibility for temperatures as high as $500 \mathrm{MeV}$, apparently reaching the onset of the dilute instanton gas approximation. Since this second aspect is at the moment under active investigation by several groups, we conclude this introduction with a brief review of the current status of topology in hot QCD.

QCD topology is an eminently nonperturbative problem which has been addressed on the lattice for a long time. It is well known that topological studies are hampered by technical difficulties on a discrete lattice $[15,16]$. However, recent methodological progress, together with more adequate computer resources, have to some extent reopened the field, leading to the first results on topology at high temperature with dynamical fermions [9,17-20]. Although these studies exhibit some common features, which we will review in the following together with our own results, a quantitative agreement has not been reached yet. Particularly significant - and still under debate-is the onset of the dilute instanton gas behavior: once this is reached, the results could be safely extrapolated to temperatures $T=\mathcal{O}(1) \mathrm{GeV}$ of cosmological relevance.

In the calculation of the topological susceptibility presented here we follow an early proposal of Kogut, Lagae, and Sinclair [21] which has also been investigated by other groups $[19,22,23]$. In a nutshell, we will use well-known identities in the fermionic sector based on the Atiyah-Singer theorem-as we will review in the following - to infer the topological susceptibility at high temperatures from the results for the chiral susceptibility.

Let us finally mention that Ref. [24] proposed to use lattice results as a quantitative input to axion cosmology. Berkowitz, Buchoff and Rinaldi [25] were the first ones to implement this suggestion in a paper which we regard as seminal, as it has inspired a large lattice activity focused on axion cosmology (despite being based on the quenched model). Not surprisingly, given the exploratory nature of these studies and the already mentioned complications of lattice topology, the results on axion cosmology have not reached a unanimous consensus: even pure Yang Mills is still under investigation (see e.g., Refs. [26,27]). This calls for further studies, thus providing one of the motivations of this work.

This paper is organised as follows. In the next section we review the lattice action and the setup of the simulations. Observables are introduced in Sec. III. The following two sections contain our results: Sec. IV is devoted to the analysis of the pseudocritical region, while in Sec. V we discuss topology and its implication on the bounds on the post-inflationary QCD axion. We close with a brief discussion on the present status of $N_{f}=2+1+1$ thermodynamics and topology, and future steps.

Some of the results presented here have been presented in a preliminary form at conferences $[10,28]$.

\section{THE ACTION AND THE SIMULATION SETUP}

We performed simulations with four flavors of maximally twisted mass Wilson fermions in the isospin limit, i.e., with degenerate, larger than physical, up and down quark masses, and physical strange and charm quark masses. In terms of the twisted fields $\chi_{l, h}=\exp \left(-i \pi \gamma_{5} \tau^{3} / 4\right) \psi_{l, h}$ the light and heavy quark twisted mass actions have the following form:

$$
\begin{aligned}
S_{f}^{l}\left[U, \chi_{l}, \bar{\chi}_{l}\right]= & \sum_{x, y} \bar{\chi}_{l}(x)\left[\delta_{x, y}-\kappa D_{\mathrm{W}}(x, y)[U]\right. \\
& \left.+2 i \kappa a \mu \gamma_{5} \delta_{x, y} \tau^{3}\right] \chi_{l}(y),
\end{aligned}
$$

and similarly

$$
\begin{aligned}
S_{f}^{h}\left[U, \chi_{h}, \bar{\chi}_{h}\right]= & \sum_{x, y} \bar{\chi}_{h}(x)\left[\delta_{x, y}-\kappa D_{W}(x, y)[U]\right. \\
& \left.+2 i \kappa a \mu_{\sigma} \gamma_{5} \delta_{x, y} \tau^{1}+2 \kappa a \mu_{\delta} \delta_{x, y} \tau^{3}\right] \chi_{h}(y),
\end{aligned}
$$


where $D_{\mathrm{W}}[U]$ is the usual Wilson operator, $a$ is the lattice spacing, and $\chi_{l, h}$ are quark spinors in the twisted basis. The hopping parameter $\kappa$ is set to its coupling-dependent critical value $\kappa_{c}(\beta)$ leading to the so-called "maximal twist" of the action (1)-(2) with the property of automatic $\mathcal{O}(a)$ improvement for expectation values of any operator [29,30]. The parameter $\mu_{l}$ describes the mass of the degenerate light quark doublet, which is still unphysically large in our study: the charged pion mass values $m_{\pi^{ \pm}}$considered at present are 210, 260,370 and $470 \mathrm{MeV}$. The heavy twisted mass parameters $\mu_{\sigma}$ and $\mu_{\delta}$ have been tuned in the unitary approach to approximately reproduce the physical $K$ and $D$ meson mass values within an accuracy of $10 \%$, thus allowing for a realistic treatment of $s$ and $c$ quarks.

For the gauge sector the Iwasaki action is used $\left(c_{0}=\right.$ 3.648 and $c_{1}=-0.331$ ):

$$
\begin{aligned}
S_{g}[U]= & \beta\left(c_{0} \sum_{P}\left[1-\frac{1}{3} \operatorname{Re} \operatorname{Tr}\left(U_{P}\right)\right]\right. \\
& \left.+c_{1} \sum_{R}\left[1-\frac{1}{3} \operatorname{Re} \operatorname{Tr}\left(U_{R}\right)\right]\right) .
\end{aligned}
$$

The two sums extend over all possible plaquettes $(P)$ and planar rectangles $(R)$, respectively.

Our finite-temperature simulations have been performed for three values of $\beta=(1.90,1.95,2.10)$. Using the nucleon mass to fix the scale, this gives $a=0.0646 \mathrm{fm}$, $a=0.0823 \mathrm{fm}$ and $a=0.0936 \mathrm{fm}$ [31]. For each lattice spacing we explored temperatures ranging from 150 to $500 \mathrm{MeV}$ by varying the temporal size of the lattice $N_{\tau}$. So far we have generated finite-temperature configurations for eight sets of parameters that correspond to four values of the charged pion mass of about 470, 370, 260 and $210 \mathrm{MeV}$, for which two, three, two and one value(s) of the lattice spacing have been considered, respectively.

For each lattice spacing the advantage of this setup ("fixed-scale approach" [32]) is that we are allowed to rely on the setup of $T=0$ simulations of the ETMC, thus exploring a wide set of temperatures. Different lattice spacings allow us to study the approach to the continuum limit. In principle, we have to deal with the mismatch of different temperatures obtained for different choices of $\beta$. In practice, the temperature scans are fine enough to overcome this potential disadvantage, as we will see when presenting the results. The full set of parameters, as well as the indicative statistics, are reported in Table I.

\section{OBSERVABLES}

We concentrate first on the study of the critical region: our primary observables here are the quasi-order parameters for deconfinement and chiral symmetry breaking: the Polyakov loop and the chiral condensate in the light sector. We also consider the disconnected chiral susceptibility: as it is merely the fluctuations of the order parameter, it has the same meaning as ordinary susceptibilities in spin models, and hence it carries the relevant information on the pseudocritical behavior. We will also discuss later how this observable acts as a proxy for the topological susceptibility in the symmetric phase.

The resulting pseudocritical temperatures characterizing the crossover (including statistical and systematical errors) are summarized in Table II.

\begin{tabular}{|c|c|c|c|c|c|}
\hline $\begin{array}{l}\text { tmft } T \neq 0 \\
\text { nomenclature }\end{array}$ & $\begin{array}{l}\text { ETMC } T=0 \\
\text { nomenclature }\end{array}$ & $m_{\pi^{ \pm}}[\mathrm{MeV}]$ & $a[\mathrm{fm}]$ & $N_{\tau} \times N_{\sigma}^{3}$ & Statistics \\
\hline D210 & D15.48 & $213(9)$ & 0.0646 & $\{4,6,8,10,12,14,16,18,20,24\} \times 48^{3}$ & $1 \mathrm{k}-7 \mathrm{k}$ \\
\hline A260 & A30.32 & 261(11) & 0.0936 & $\begin{array}{c}\{4,6,8,10,11,12,14\} \times 32^{3} \\
\{16\} \times 40^{3} \\
\{20\} \times 48^{3}\end{array}$ & $\begin{array}{c}1 \mathrm{k}-5 \mathrm{k} \\
3 \mathrm{k} \\
4 \mathrm{k}\end{array}$ \\
\hline B260 & B25.32 & $256(12)$ & 0.0823 & $\{4,5,6,8,10,12,14,16,18\} \times 40^{3}$ & $1 \mathrm{k}-8 \mathrm{k}$ \\
\hline A370 & A 60.24 & $364(15)$ & 0.0936 & $\begin{array}{c}\{3,4,5,6,7,8,9,10,11,12\} \times 24^{3} \\
\{13,14\} \times 32^{3}\end{array}$ & $\begin{array}{l}2 \mathrm{k}-9 \mathrm{k} \\
5 \mathrm{k}, 27 \mathrm{k}\end{array}$ \\
\hline $\begin{array}{l}\text { B370 } \\
\text { B370.24 }\end{array}$ & B55.32 & $372(17)$ & 0.0823 & $\begin{array}{c}\{3,4,5,6,7,8,10,11,12,13,14,15,16\} \times 32^{3} \\
\{4,6,8,10,11,12\} \times 24^{3}\end{array}$ & $\begin{array}{l}2 \mathrm{k}-10 \mathrm{k} \\
3 \mathrm{k}-10 \mathrm{k}\end{array}$ \\
\hline D370 & D45.32 & $369(15)$ & 0.0646 & $\begin{array}{c}\{5,6,7,8,9,10,12,14,16\} \times 32^{3} \\
\{18\} \times 40^{3} \\
\{20\} \times 48^{3}\end{array}$ & $\begin{array}{c}1 \mathrm{k}-12 \mathrm{k} \\
10 \mathrm{k} \\
10 \mathrm{k}\end{array}$ \\
\hline A470 & $\mathrm{A} 100.24 \mathrm{~s}$ & $466(19)$ & 0.0936 & $\begin{array}{c}\{4,5,6,7,8,9,10,11,12\} \times 24^{3} \\
\{14\} \times 32^{3}\end{array}$ & $\begin{array}{c}3 \mathrm{k}-8 \mathrm{k} \\
8 \mathrm{k}\end{array}$ \\
\hline B470 & B85.24 & $465(21)$ & 0.0823 & $\begin{array}{c}\{4,5,6,7,8,9,10,11,12\} \times 24^{3} \\
\{13,14\} \times 32^{3}\end{array}$ & $\begin{array}{r}2 \mathrm{k}-4 \mathrm{k} \\
2.5 \mathrm{k}, 7 \mathrm{k} \\
\end{array}$ \\
\hline
\end{tabular}

TABLE I. $\quad T=0$ base ensembles, charged pion mass, lattice spacings and finite-temperature ensembles generated so far. 
TABLE II. Summary of fit-estimated pseudocritical temperatures using fermionic and gluonic observables. The first error is statistical, while the second is systematic; see text for details

\begin{tabular}{lccccccc}
\hline \hline Ensemble & $a[\mathrm{fm}]$ & $m_{\pi}[\mathrm{MeV}]$ & $T_{\chi}[\mathrm{MeV}]$ & $T_{\Delta}[\mathrm{MeV}]$ & $T_{\Delta}^{p}[\mathrm{MeV}]$ & $T_{\text {deconf }}[\mathrm{MeV}]$ & $T_{\text {deconf }}^{\mathcal{F}}[\mathrm{MeV}]$ \\
\hline D210 & 0.065 & 213 & $158(1)(4)$ & $165(3)(1)$ & $161(2)(8)$ & $176(8)(8)$ & $1.2(6)(1)$ \\
A260 & 0.094 & 261 & $157(8)(14)$ & $172(2)(1)$ & $186(3)(8)$ & $188(1)(1)(1)$ \\
B260 & 0.082 & 256 & $161(13)(2)$ & $177(2)(1)$ & $181(1)(9)$ & $192(9)(2)$ & $196(13)(1)$ \\
A370 & 0.094 & 364 & $185(5)(3)$ & $191(2)(0)$ & $202(1)(10)$ & $202(1)(8)$ & $192(12)(15)$ \\
B370 & 0.082 & 372 & $189(2)(1)$ & $194(2)(0)$ & $196(1)(8)$ & $201(6)(0)$ & $181(12)(6)$ \\
D370 & 0.065 & 369 & $185(1)(3)$ & $180(5)(1)$ & $188(2)(9)$ & $193(13)(2)$ & $196(65)(20)$ \\
A470 & 0.094 & 466 & $200(4)(6)$ & $193(5)(2)$ & $204(5)(10)$ & $205(4)(2)$ & $184(15)(10)$ \\
B470 & 0.082 & 465 & $203(2)(2)$ & $202(7)(1)$ & $204(2)(10)$ & $212(6)(1)$ & $193(17)(14)$ \\
\hline \hline
\end{tabular}

\section{A. Polyakov loop}

The Polyakov loop is defined as a Wilson loop of gauge fields winding once around the temporal (thermal) direction. The important quasi-order parameter is the real part of it,

$$
\operatorname{Re}(L)=\frac{1}{N_{c}} \frac{1}{N_{\sigma}^{3}} \operatorname{Re} \operatorname{Tr} \sum_{\mathbf{x}} \prod_{x_{4}=0}^{N_{\tau}-1} U_{4}\left(\mathbf{x}, x_{4}\right)
$$

We may only consider the real part since when quarks are present the center symmetry is explicitly broken towards the real sector. In pure gauge theory this quantity is strictly the order parameter for the deconfinement transition, and since the pure gauge limit is approached for large quark masses the Polyakov loop also acts as a quasi order parameter with dynamical quarks. It may be renormalized as follows [33]:

$$
\langle\operatorname{Re}(L)\rangle_{R}=\langle\operatorname{Re}(L)\rangle \exp \left(V\left(r_{0}\right) / 2 T\right)
$$

where $V\left(r_{0}\right)$ denotes the static quark-antiquark potential at the distance of the Sommer scale $r=r_{0}$ [34]. The latter is to be determined at zero temperature. The static quarkantiquark potential $V(r)$ has been evaluated using 20 steps of APE smearing with a smearing parameter $\alpha_{\mathrm{APE}}=0.5$. For $r_{0}$ we use the values determined by the ETMC. Subsequently $V(r)$ was interpolated using cubic splines and its value $V\left(r_{0}\right)$ has been extracted.

The results for the potential are summarized in Fig. 1 and the extraction of the value $V\left(r_{0}\right)$ needed for the renormalization of the Polyakov loop is exemplified for the results for a pion mass of $210 \mathrm{MeV}$ on the $\mathrm{D}$ ensembles. The others are completely analogous. It is important to note that this prescription [33] introduces a further dependence on temperature; hence in the crossover region we should not expect that the results for the pseudocritical temperatures match those obtained using other temperatureindependent renormalization prescriptions. Of course in the infinite-mass limit, when the Polyakov loop becomes an exact order parameter for deconfinement, these ambiguities disappear.

\section{B. Chiral condensate}

The chiral condensate $\langle\bar{\psi} \psi\rangle$ in the light sector serves as an (approximate) order parameter for the $S U(2) \times S U(2)$ symmetry, which is explicitly broken by the quark mass:

$$
\langle\bar{\psi} \psi\rangle=\frac{T}{V} \frac{\partial \ln Z}{\partial m_{q}}=\frac{1}{N_{\sigma}^{3} N_{\tau}}\left\langle\operatorname{Tr} M_{q}^{-1}\right\rangle .
$$

The trace of the inverse is evaluated by means of the technique of noisy estimators using multiple quark matrix inversions acting on 24 Gaussian noise vectors. At maximal twist the leading ultraviolet-divergent part is proportional to $\mu / a^{2}$ which in our early $N_{f}=2$ study was removed by subtracting the corresponding condensate at the same mass at $T=0$ [35]. The multiplicative renormalization factors are then canceled by dividing by the condensate at zero temperature in the chiral limit:

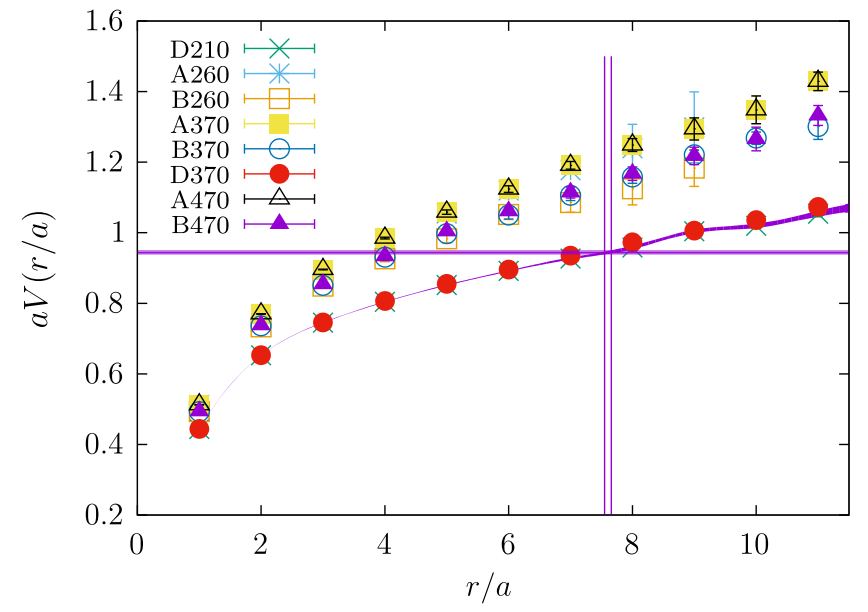

FIG. 1. The results for the potential at zero temperature used for the renormalization of the Polyakov loop. The vertical band is drawn in correspondence with the value of $r_{0} / a$ calculated by the ETMC for the D ensemble and a pion mass of $210 \mathrm{MeV}$; hence the horizontal band indicates the corresponding renormalization parameter $V_{0}$ which has been numerically determined as described in the main text. Analogous procedures have been followed for the other ensembles. 


$$
R_{\langle\bar{\psi} \psi\rangle}=\frac{\langle\bar{\psi} \psi\rangle\rangle_{l}^{T, \mu}-\langle\bar{\psi} \psi\rangle_{l}^{T=0, \mu}+\langle\bar{\psi} \psi\rangle_{l}^{T=0, \mu=0}}{\langle\bar{\psi} \psi\rangle_{l}^{T=0, \mu=0}} .
$$

Having now the strange quark at our disposal, we use another prescription based on a suitable subtraction (involving the light and strange masses) of the strange quark condensate. It eliminates the additive $\mu$-proportional divergence contained in the light condensate being estimated by means of the strange quark condensate. This procedure has been proposed for $N_{f}=2+1$ flavors in the literature [36]:

$$
\Delta_{l, s}=\frac{\langle\bar{\psi} \psi\rangle_{l}-\frac{\mu}{\mu_{s}}\langle\bar{\psi} \psi\rangle_{s}}{\langle\bar{\psi} \psi\rangle_{l}^{T=0}-\frac{\mu}{\mu_{s}}\langle\bar{\psi} \psi\rangle_{s}^{T=0}},
$$

where $\langle\bar{\psi} \psi\rangle_{s}$ is the strange quark condensate calculated in the Osterwalder-Seiler setup $[29,37]$ to avoid mixing in the heavy quark sector and where the strange mass $\mu_{s}$ has been determined as to reproduce the physical mass of $\bar{s} \gamma_{\mu}$ s. The corresponding expression at $T=0$ serves as a normalization factor.

\section{Chiral susceptibility}

The chiral susceptibility, defined as the mass derivative of the chiral condensate reads

$$
\chi=\frac{V}{T} \frac{\partial}{\partial m_{l}}\langle\bar{\psi} \psi\rangle_{l} \equiv \chi_{\bar{\psi} \psi \psi}^{\mathrm{disc}}+\chi_{\bar{\psi} \psi}^{\mathrm{conn}} .
$$

The disconnected chiral susceptibility, which carries the relevant information on the critical behavior, is the quadratic fluctuation of the chiral condensate,

$$
\chi_{\bar{\psi} \psi \psi}^{\mathrm{disc}}=\frac{V}{T}\left(\left\langle(\bar{\psi} \psi)^{2}\right\rangle_{l}-\langle\bar{\psi} \psi\rangle_{l}^{2}\right),
$$

for which the traces arising from the path integral have been evaluated using the stochastic noise method and we made sure that no net connected piece enters the final result. Ultraviolet divergences cancel in the difference, and we are left only with a multiplicative renormalization.

\section{THERMAL TRANSITION TEMPERATURE(S)}

We have considered the renormalized Polyakov loop and two chiral observables in order to extract three different pseudocritical temperatures. We will discuss the used fit strategies in what follows.

\section{A. From the Polyakov loop}

The deconfinement transition temperature $T_{\text {deconf }}$ is read off from the inflection point of a hyperbolic tangent function fit to the renormalized Polyakov loop data

$$
\langle\operatorname{Re}(L)\rangle_{R}=A_{P}+B_{P} \tanh \left(C_{P}\left(T-T_{\text {deconf }}\right)\right) .
$$

The data becomes more and more noisy for larger $N_{\tau}$ which mostly reduces the data quality for the small mass ensembles. The data and relevant hyperbolic tangent fits for the renormalized Polyakov loop are shown in Fig. 2.

We have restricted the fits to temperatures below $T=$ $310 \mathrm{MeV}$ for our central fits in order to avoid the region of visible discretization artifacts at high temperatures. We have always included in the fits all available data at the lowtemperature end since data is very limited there.

A second fit was performed including higher-temperature data up to $T=380 \mathrm{MeV}$ (or up to $T=400 \mathrm{MeV}$ in the case of B260, respectively) in order to estimate a systematic error for $T_{\text {deconf }}$ taking half of the deviation from the central fit results above.

In the middle panel of Fig. 2 we have added the Polyakov loop data of our finite-size test $24^{3}$ ensemble B370.24. There is no difference in the data as compared to the data obtained in the larger volume $32^{3}$.

As we have already discussed at the end of Sec. III A, this renormalization prescription is temperature dependent and
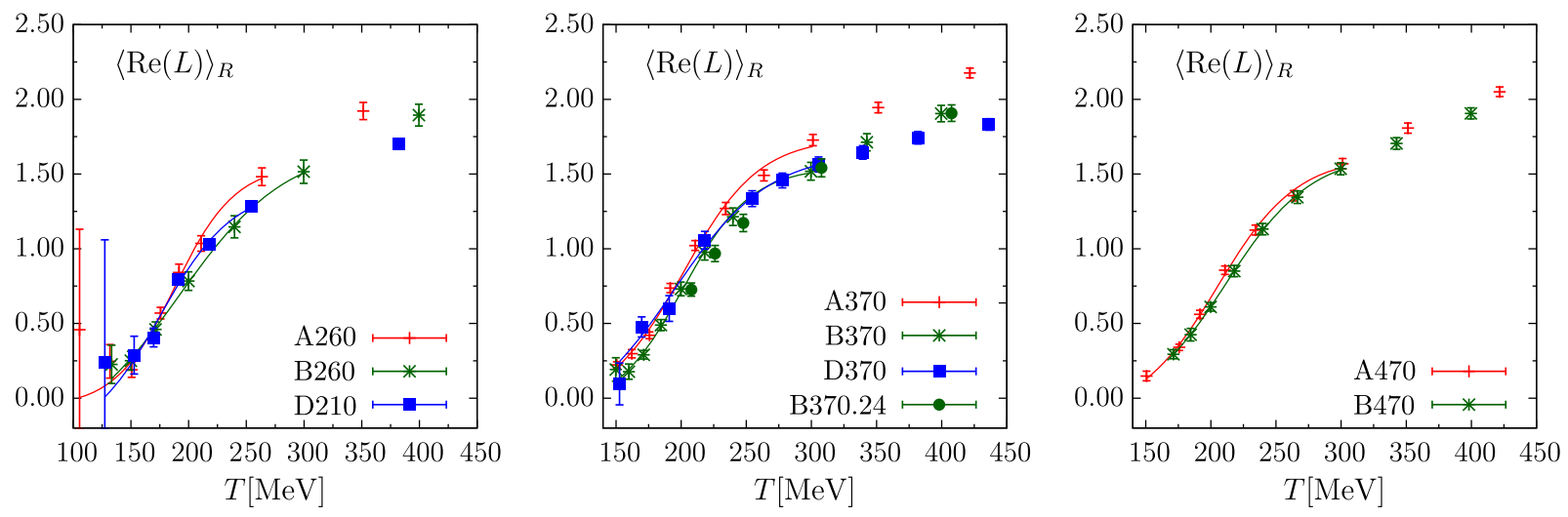

FIG. 2. The renormalized Polyakov loop. Left: For $m_{\pi}=210 \mathrm{MeV}$ (blue points) and for $m_{\pi}=260 \mathrm{MeV}$. Middle: For $m_{\pi}=370 \mathrm{MeV}$. The data of the finite-size test ensemble B370.24 is shown slightly shifted for ease of reading. Right: For $m_{\pi}=470 \mathrm{MeV}$. 

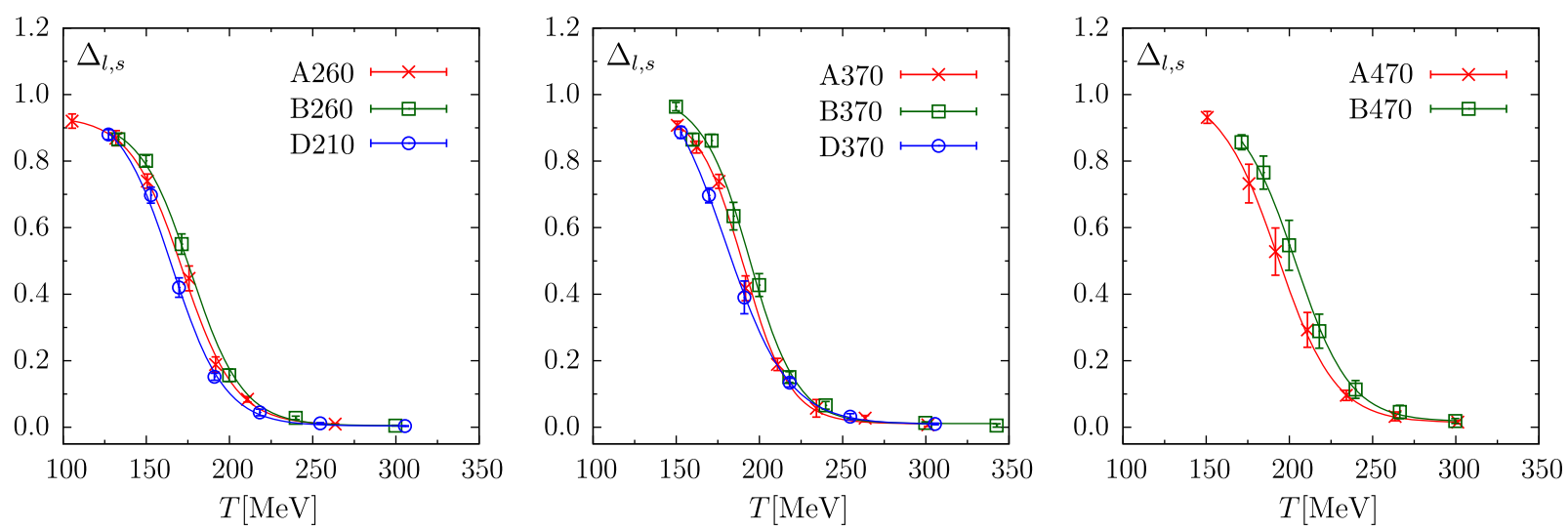

FIG. 3. The renormalized chiral condensate $\Delta_{l s}$. Left: For $m_{\pi}=210 \mathrm{MeV}$ (blue points) and for $m_{\pi}=260 \mathrm{MeV}$. Middle: For $m_{\pi}=370 \mathrm{MeV}$. Right: For $m_{\pi}=470 \mathrm{MeV}$.

hence it affects the estimate of the pseudocritical temperature in the crossover region. This is just one of the many examples of a pseudocritical temperature which is not unique. As an alternative estimate of the pseudocritical temperature for deconfinement we may define $T_{\text {deconf }}^{\mathcal{F}}$ as the inflection point of the free energy for a static quark (or, equivalently, from the peak of the entropy for a static quark). Consider the free energy $\mathcal{F}=-T \log \operatorname{Re}(L)_{R}$ where the renormalization constant is only additive and does not influence the inflection point position. We have performed hyperbolic tangent fits in the interval [130:300] MeV and we have estimated the systematic error on the inflection point by varying the fit interval and/or the fit function (polynomial fits). As it is well known, the free energy is rather smooth (due to the logarithm); for the lightest mass the fits were not successful and in the other cases the identification of the inflection point has large errors. All in all $T_{\text {deconf }}^{\mathcal{F}}$ is consistent with $T_{\text {deconf }}$ within the errors. A high-statistics study with a finer range of temperature will be necessary to reveal the expected shift between $T_{\text {deconf }}$ and $T_{\text {deconf }}^{\mathcal{F}}$ which will anyway disappear when the Polyakov loop is an exact order parameter.

\section{B. From the renormalized chiral condensate}

In Fig. 3 , the first estimate $T_{\Delta}$ for the crossover temperature $T_{c}$ is determined from the renormalized chiral condensate. The data of $\Delta_{l s}$ follows an S-shape curve for all considered ensembles. We therefore used as a fit ansatz the following sigmoid function:

$$
\Delta_{l s}(T)=A_{\Delta}+B_{\Delta} \tanh \left(-C_{\Delta}\left(T-T_{\Delta}\right)\right) .
$$

We always used all available data at low temperatures and used two upper limits for the fit ranges in temperature. The main fits were obtained with data with $T<350 \mathrm{MeV}$ and another extended fit has been done with $T<450 \mathrm{MeV}$. Half the deviation of the latter from the main fit results was used to estimate the systematic error.
Second, we have used a simple polynomial fit

$$
\Delta_{l s}(T)=a_{\Delta}+b_{\Delta} T+c_{\Delta} T^{2}+d_{\Delta} T^{3} .
$$

We started from an exact fit using four points and we progressively enlarged the interval until the quality of the fit deteriorated. The inflection point is then $T_{\Delta}^{p}=-c_{\Delta} /\left(3 d_{\Delta}\right)$ and one first estimate of the error is given by the maximum between the statistical errors of the individual fits, and the dispersion of the results obtained by changing the interval as described. Taking into account that the polynomial fit merely serves as an interpolator in the given interval, a second more conservative estimate for the error uses the resolution of the interpolation; in practice, half of the distance between the simulation points which are closest to $T_{\Delta}$. We quote both errors in the table, where we also keep both results, from the sigmoid fit and from the polynomial fit, as the dispersion between them offers a further estimate of the errors. It turns out that within the larger errors associated with the polynomial fits $T_{\Delta}$ and $T_{\Delta}^{p}$ agree.

\section{From $T \chi_{\bar{\psi} \tilde{\mu} \psi}^{\text {disc }}$}

Here the second estimate for the crossover temperature $T_{\chi}$ is determined from the chiral susceptibility. We noted that by considering the $T \chi_{\bar{\psi} \psi}^{\mathrm{disc}}$ rather than $\chi_{\bar{\psi} \mu \psi}^{\mathrm{disc}}$ the signal is sharper, and we used this in our estimates. The modest shift in the pseudocritical temperature associated with this different normalization is about $10 \mathrm{MeV}$, which is negligible with respect to other errors.

For estimating the position of the peak in the (modified) disconnected chiral susceptibility (10) we fit an ansatz quadratic in the temperature to the data:

$$
\chi_{\bar{\psi} \mu \psi}^{\mathrm{disc}}(T)=A_{\chi}+B_{\chi}\left(T-T_{\chi}\right)^{2},
$$

a definition that does not assume a model of the $T$ dependence of the susceptibility data but is a generic fit function that should be valid in the close vicinity of a peak. 
We have fitted it to the peak regions of our $\chi_{\bar{\psi} \psi}^{\text {disc }}$ data. Each ensemble was fitted separately and we have considered several fit regions in temperature for each ensemble to address the systematic uncertainty of its choice. We take the central values and the statistical errors from the bestfit result (in terms of $\chi^{2} /$ d.o.f.) and use fits with $\chi^{2} /$ d.o.f. $<2.5$ or with $\chi^{2} /$ d.o.f. $=\infty$ (i.e., the parameters are fully constrained by the data). Among the allowed fit results imposing the just-mentioned conditions on the fit quality we picked the one with the maximum deviation from the central fit and took half of the deviation as the systematic error.

Since we adopted a fixed-scale approach the number of data points we are able to measure in the peak region is restricted by construction. It is even more restricted for the smaller pion masses since there intermediate points would be at large odd $N_{\tau}$ which are prohibitively expensive in terms of computing time and therefore out of the reach of our present capabilities. Consequently, the peak region for the small pion masses is not too well covered by data and the fits turned out to be more difficult than those to the renormalized quark condensate discussed previously.
In Figs. 4-6 we show for each ensemble the best fit obtained upon varying the fit range in $T$.

Figure 4 shows the chiral susceptibility for the three ensembles with the lightest pion. While the peak is nicely visible for the ensemble at a pion mass of $\sim 210 \mathrm{MeV}$ the maximum of the data is much more weakly pronounced for the two ensembles at a pion mass of about $260 \mathrm{MeV}$ which in both cases is mainly due to the smallest temperature points. While for the A260 ensemble the best fit is accordingly found by discarding the smallest temperature point, the fits for the B260 ensemble favor including it.

\section{The pseudocritical line in the temperature-mass plane}

Figure 7 shows the obtained results for $T_{\chi}$ and $T_{\Delta}$. The estimates of $T_{\chi}$ and $T_{\Delta}$ at physical quark masses were obtained by the Wuppertal-Budapest Collaboration [12], with staggered quark discretizations. There is also the HotQCD result from an independent study with chiral domain wall fermions [14], which is almost identical to the value from their staggered analysis [13].
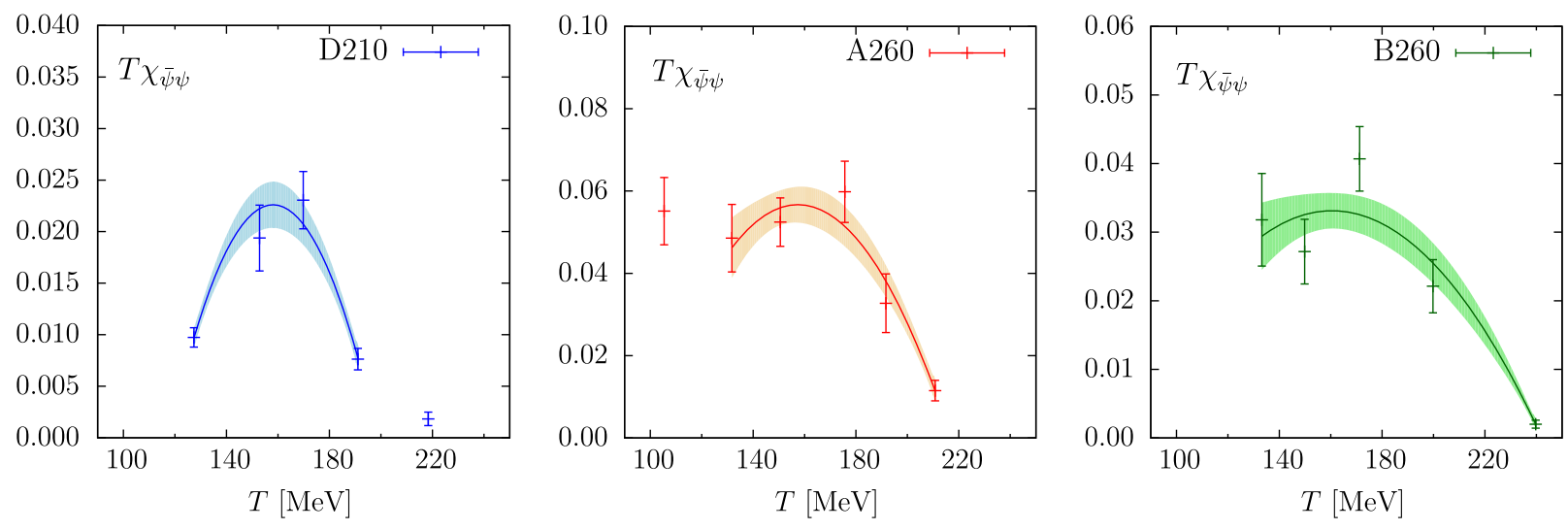

FIG. 4. Bare disconnected chiral susceptibility $T \chi_{\bar{\psi} \psi}^{\text {disc }}$ and fits for different ensembles, for the two lightest pion masses, and different lattice spacings. Left: D210. Middle: A260. Right: B260 MeV. The best fit to the peak area for the respective ensemble is shown as a smooth curve together with the corresponding error envelope.
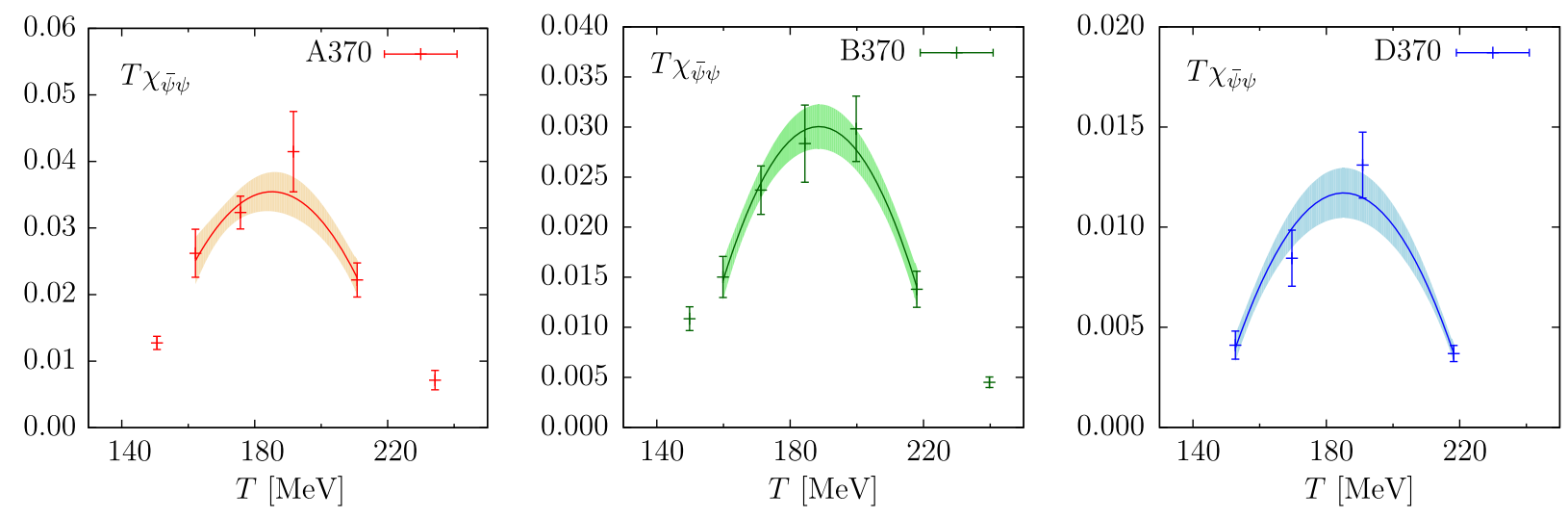

FIG. 5. Same as in Fig. 4, but for a pion mass of 370 MeV. Left: A370. Middle: B370. Right: D370 MeV. 

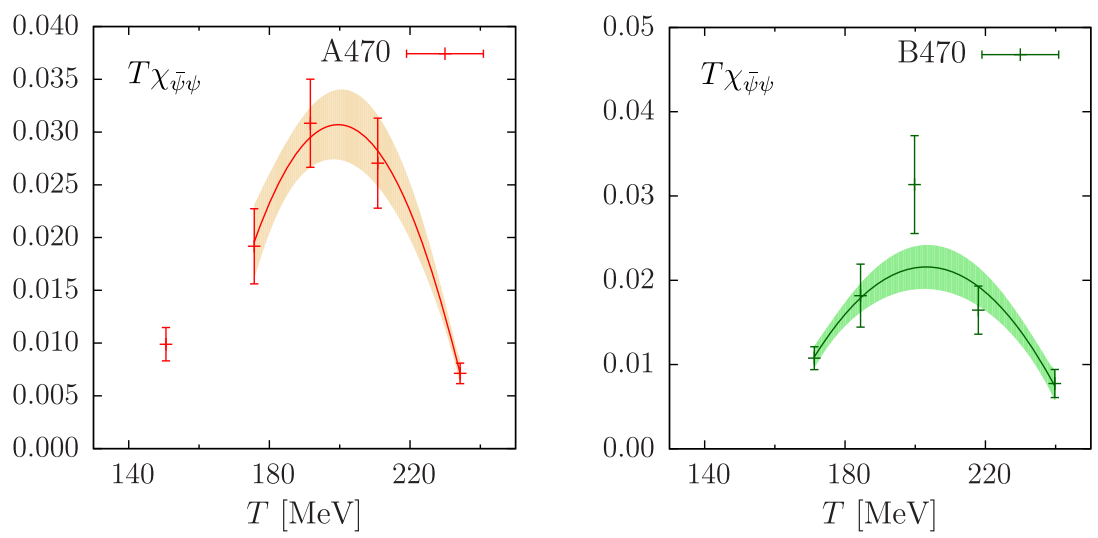

FIG. 6. Same as in Fig. 4, but for the heaviest pion mass $470 \mathrm{MeV}$. Left: A470. Right: B470 MeV.

We fitted our values of $T_{\chi}$ for the two scenarios discussed above in the context of the order of the phase transition in the chiral limit of the two-flavor theory $[2,4]$. First, we confirm that for these relatively large masses we cannot discriminate between different critical scenarios. On the positive side, we note that either plausible parametrization interpolates the data, and fares well regarding the results for physical pion masses obtained with $N_{f}=2+1$ staggered and domain wall fermions. We thus cross-check the results for the pseudocritical temperature provided by other groups, and, at the same time, confirm that a dynamical charm does not contribute to the chiral dynamics in the pseudocritical region; see Fig. 7.

We close by noting two features of our results which call for further investigations. In the $O(4)$ scaling window we

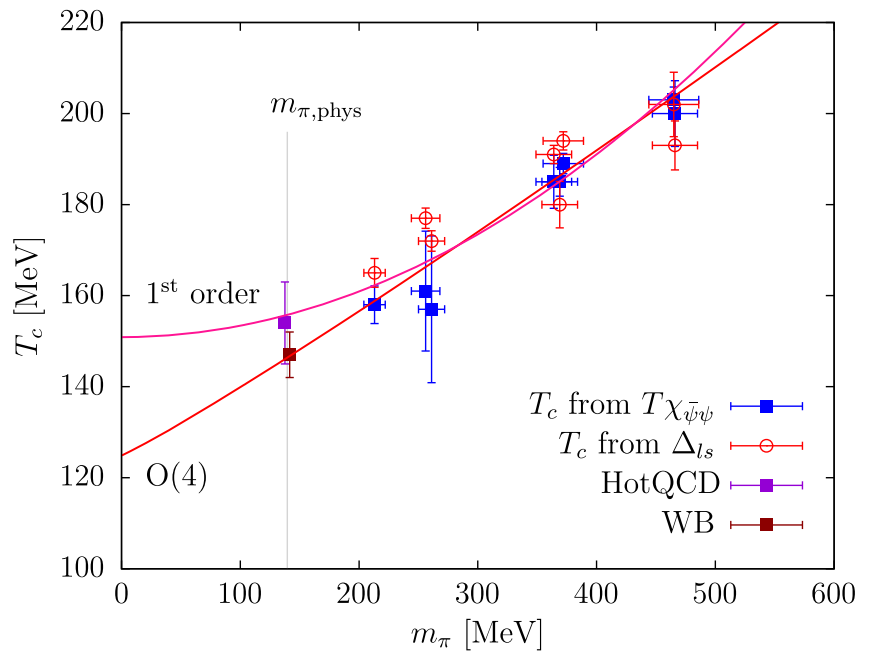

FIG. 7. Pseudocritical temperature as a function of the pion mass, superimposed by the curves corresponding to first- and second-order chiral scenarios. The staggered value from the Wuppertal-Budapest Collaboration [12] and the coinciding result from both staggered [13] and domain wall [14] studies of the HotQCD Collaboration for physical pion mass are shown as well. Data from the disconnected chiral susceptibility $T \chi \chi_{\bar{\psi} \psi \psi}^{\text {disc }}$ have been used in the fits. See text for discussions. would expect $\left(T \chi_{\bar{\psi} \mu \mu}^{\mathrm{disc}}-T_{0}\right) /\left(T_{\Delta}-T_{0}\right) \simeq 1.5$, which is violated by our results once we assume $T_{0} \simeq 138 \mathrm{MeV}$. It is an open question whether this is a correction to $O(4)$ scaling, or if indeed the $O(4)$ scenario should be abandoned, or $T_{0}$ should be revised. Also, again in contrast to scaling expectations, the two chiral temperatures seem to get closer to each other at large mass. This effect may be due to a statistical fluctuation at $m_{\pi}=260 \mathrm{MeV}$ (if we discard this point the trend, which is anyway small, disappears), or, again, to large mass scaling violations. Note that also the Polyakov loop pseudocritical temperature gets closer to $T_{\Delta}$ at larger pion masses, which may indeed indicate the onset of the heavy quark regime. Obviously the validity of the chiral scaling, and the crossover to the heavy quark regime are interesting questions but beyond the scope of the present study. We hope to be able to return to them in a future investigation.

\section{TOPOLOGY AND AXION'S PROPERTIES}

Let us first briefly recall how axion physics and topology are intimately connected. We will then describe the results for the topological susceptibility, comment on the scaling with the pion mass, and discuss the implications on the post-inflationary axion's mass bound.

The QCD Lagrangian admits a $C P$-violating term

$$
\mathcal{L}=\mathcal{L}_{\mathrm{QCD}}+\theta \frac{g^{2}}{32 \pi^{2}} F_{\mu \nu}^{a} \tilde{F}_{a}^{\mu \nu}
$$

where we recognize that $\frac{g^{2}}{32 \pi^{2}} F_{\mu \nu}^{a} \tilde{F}_{a}^{\mu \nu}$ is the topological charge density $Q(x)$. The $\theta$ term gives an electric dipole moment to the neutron, which is strongly constrained experimentally [38] leading to the bound $\theta<10^{-10}$. The strong $C P$ problem consists in explaining this unnaturally small value.

An elegant solution to the strong $C P$ problem postulates the existence of an extra particle [39-41], a pseudoGoldstone boson of the spontaneously broken PecceiQuinn symmetry, which couples to the QCD topological charge, with a coupling suppressed by a scale $f_{a}$. The thermal grand canonical partition function of QCD is now a function of $\theta$ and the temperature $T$, 

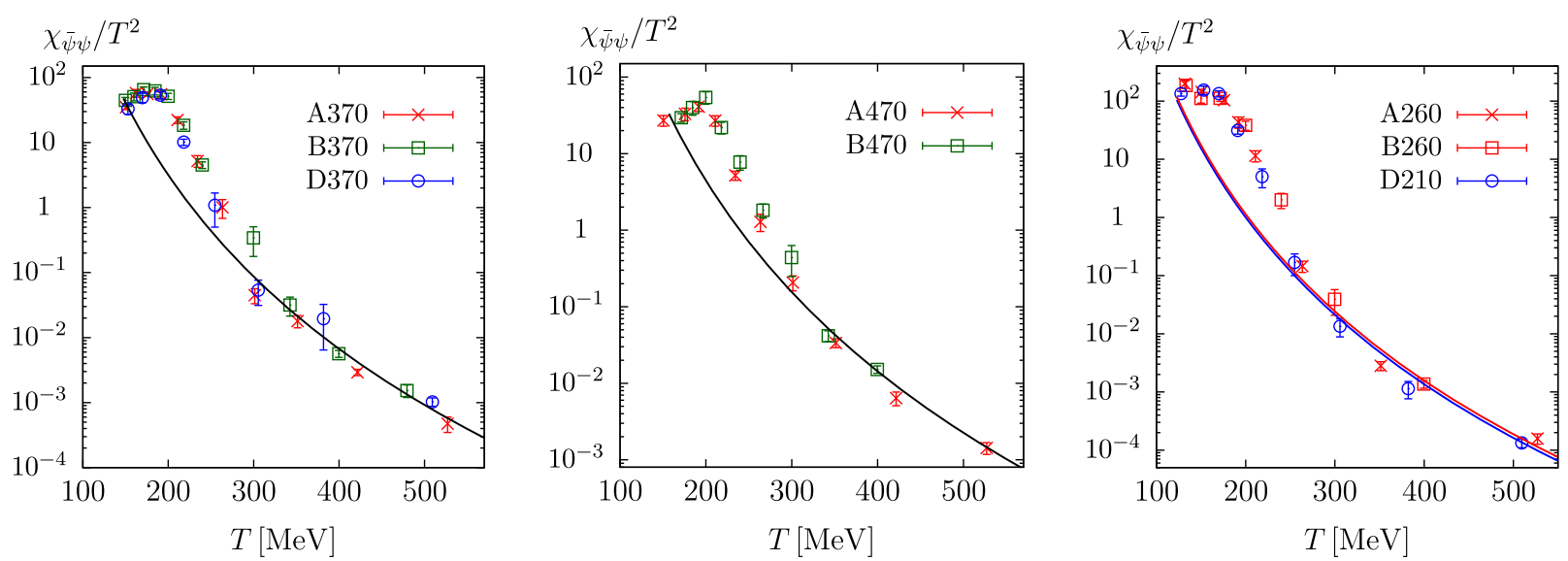

FIG. 8. The chiral disconnected susceptibility, evaluated on the different ensembles corresponding to pion masses ranging from 210 to $470 \mathrm{MeV}$, and on lattices of different coarseness. For each pion mass the residual lattice spacing dependence is below the statistical errors. We superimpose the central results of the fits to the simple power-law falloff described in the text.

$\mathcal{Z}_{\mathrm{QCD}}(\theta, T)=\int \mathcal{D}[\Phi] e^{-T \sum_{t} \int d^{3} x \mathcal{L}_{\mathrm{QCD}}(\theta)}=e^{-V F(\theta, T)}$

and the free energy $F(\theta, T)$ is the axion potential.

At leading order in $1 / f_{a}$ (well justified as $f_{a} \gtrsim 4 \times 10^{8} \mathrm{GeV}$ ) the axion can be treated as an external source, and its mass is given by

$$
m_{a}^{2}(T) f_{a}^{2}=\left.\frac{\partial^{2} F(\theta, T)}{\partial \theta^{2}}\right|_{\theta=0} \equiv \chi_{\mathrm{top}}(T)
$$

The cumulants $C_{n}$ of the topological charge distribution are related to the Taylor coefficients of the expansion of the free energy around $\theta=0$

$$
F(\theta, T)=V \sum_{n=1}^{\infty}(-1)^{n+1} \frac{\theta^{2 n}}{(2 n) !} C_{n},
$$

and hence higher-order cumulants and their ratios carry information on the axion's interactions. We will consider higher-order cumulants in a companion paper based on the gluonic measurements [17,42], while in this paper we will use our results on topological susceptibility exclusively to constrain the (post-inflationary) axion mass.

\section{A. Topological susceptibility}

We measure the topological susceptibility following Refs. $[19,21,22]$. They started from the continuum identity

$$
Q(x)=\frac{g^{2}}{32 \pi^{2}} F_{\mu \nu}^{a} \tilde{F}_{a}^{\rho \sigma}=m_{l} \int d^{4} x \bar{\psi}(x) \gamma_{5} \psi(x)
$$

and noted that this remains true on a smooth gauge field configuration (i.e., if lattice artifacts are small); hence, by squaring Eq. (19),

$$
\chi_{\text {top }} \equiv \frac{\left\langle Q^{2}\right\rangle}{V}=m_{l}^{2} \chi_{5}^{\text {disc }} .
$$

It is well known that $\chi_{5}^{\text {disc }}$ suffers from huge fluctuations. Rather than attempting its measurement, one considers that when chiral symmetry is restored, $\chi_{5}^{\mathrm{disc}} \approx \chi_{\overline{\bar{\psi}} \psi}^{\mathrm{disc}}$.

The main quantity of interest for our topological analysis thus turns out to be the disconnected susceptibility of the chiral condensate, $\chi_{\bar{\psi} \psi}^{\mathrm{disc}}=\frac{N_{\sigma}^{3}}{T}\left(\left\langle(\bar{\psi} \psi)^{2}\right\rangle_{l}-\langle\bar{\psi} \psi\rangle_{l}^{2}\right)$ which we have already discussed within the pseudocritical region. For our topological study we will consider results in the extended temperature range, and based on these results we evaluate the topological susceptibility which we show in Fig. 8. We have grouped the results according their mass value, and in each plot we show results for the different lattice spacings. Within our statistical errors we cannot see any lattice spacing dependence, and we will take our results as continuum estimates. In the next subsection we comment in more detail on scaling and the continuum limit.

In Fig. 9 we show all the results for the topological susceptibility on a log-log scale in the high-temperature

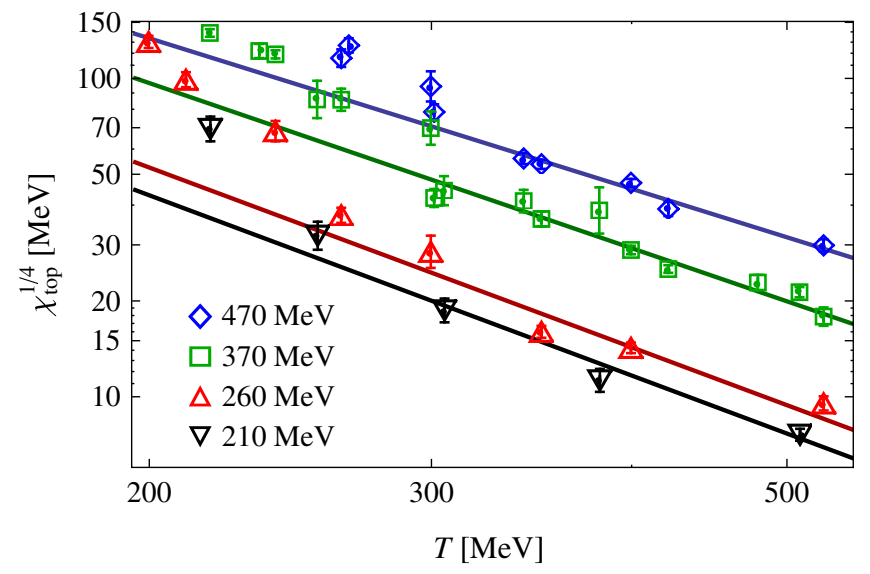

FIG. 9. The topological susceptibility from the chiral disconnected susceptibility, for different masses at all available lattice spacings. We superimpose power-law fits $\chi_{\text {top }} \simeq A T^{-d}$ described in the text. 

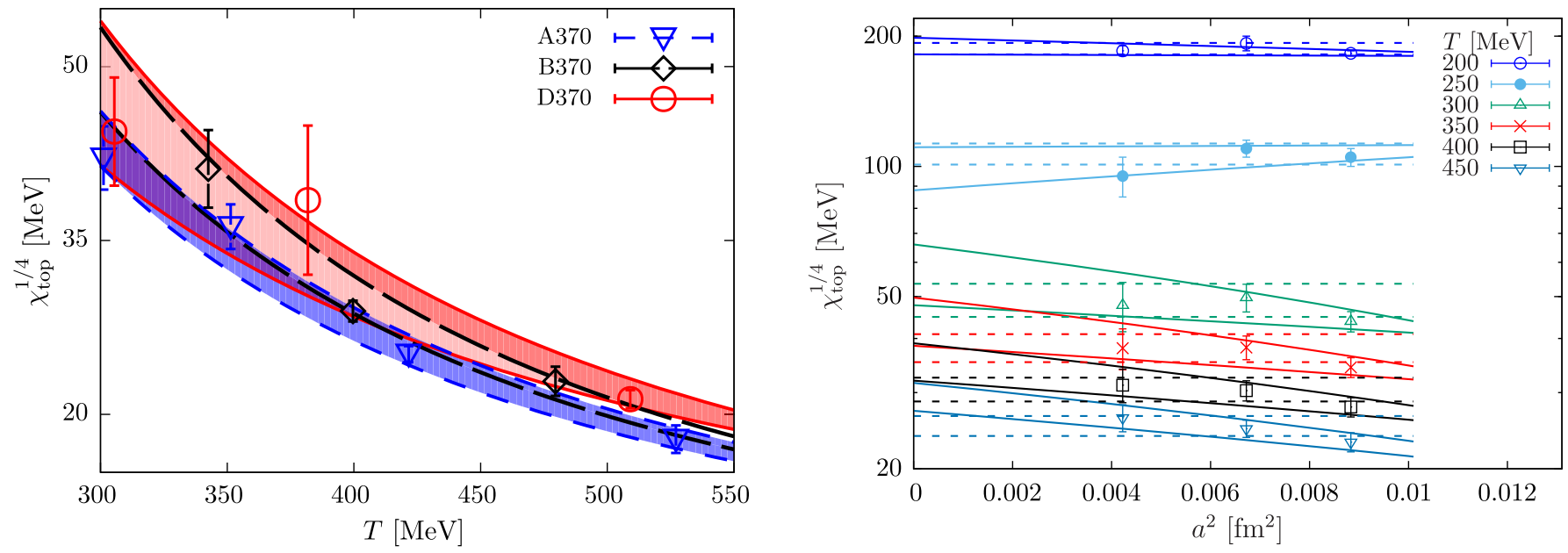

FIG. 10. Left: The topological susceptibility computed on $m_{\pi}=370 \mathrm{MeV}$ lattices with different spacings. Power-law fits for individual ensembles are superimposed, together with their error bands. Right: Pointwise continuum extrapolations for interpolated $\chi_{\text {top }}$. The interpolations are needed to match temperatures for results obtained at different lattice spacings using power-law fits (as shown in the left panel) for $T>300 \mathrm{MeV}$ and a polynomial interpolation at lower temperatures. The dashed and solid lines correspond to zerothand first-order extrapolations in $a^{2}$, respectively, as described in the text.

region. We superimpose the fits to a simple power-law falloff $\chi_{\text {top }}(T)=A T^{-d}$, which describe the data for $T>350 \mathrm{MeV}$, with a power roughly independent of the mass. As already stated, we could not detect any lattice spacing dependence within our accuracy, and hence we globally fit all data belonging to the same mass for all values of lattice spacing.

The fit parameters $A$ and $d$ are strongly correlated and fit errors have little meaning; we merely quote the central values for $d=(6.26,6.88,7.52,7.48)$ for decreasing values of the mass $m_{\pi}=(470,370,260,210) \mathrm{MeV}$. To get a feeling for the error of the exponent $d$ we may explore the functional dependence of the topological susceptibility on the temperature by defining a local effective power [43] $d_{\text {eff }}(T)=T d \log \chi_{\text {top }}(T) / d T$ which we show in Fig. 11. In

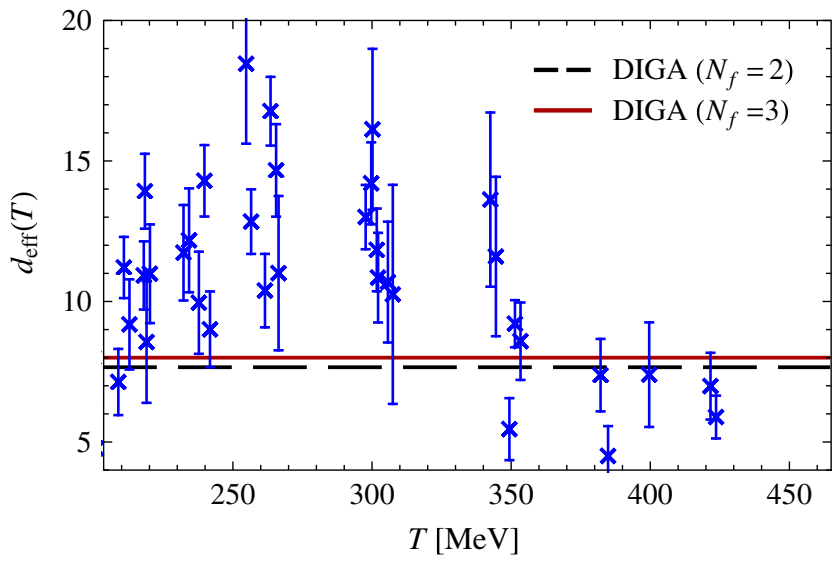

FIG. 11. The effective exponent describing the (local) powerlaw behavior of the topological susceptibility. All available pion masses and lattice spacings are used in the plot. The results from the dilute instanton gas are shown as well. doing so, we do not distinguish different masses and spacings: as the log derivative is rather noisy, it is not possible to detect any trend with mass and spacing and we simply plot all the results together. The average result is broadly consistent with the fits, and apparently approaches a constant value above $T \sim 350 \mathrm{MeV}$, while the spread of the exponents could be taken as an estimate of the error on the power $d$ of the falloff.

Concerning the apparent constant asymptotic behavior, we expect that at very large temperatures the partition function is described by a dilute gas of instantons and anti-instantons (DIGA), which leads to a well-defined prediction for the falloff of the topological susceptibility, also shown in Fig. 11. Apparently the DIGA result is approached already for these temperatures; however, it is not clear from the plot whether such a decreasing trend with temperature has reached its nearly asymptotic value (modulo small corrections), or, rather, if the apparent coincidence with the DIGA value is accidental, and restricted to a limited range of temperatures. Only simulations for larger temperatures can settle this issue.

Further, we note that the DIGA result is approached from above: the exponent has larger values for smaller temperatures; see again Fig. 9. Very interestingly, it has recently been proposed [44] that close to $T_{c}$ the dilute instanton gas changes to an ensemble of instantons and dyons, and the signature for this should be a faster decrease of the topological susceptibility close to $T_{c}$.

\section{B. Details on lattice artifacts}

We focus on the results for $m_{\pi}=370 \mathrm{MeV}$, where we have results at the three lattice spacings. First, we performed individual power-law fits that we are going to use as 
interpolators for $T>300 \mathrm{MeV}$. We display the fits for $m_{l}^{2} \chi_{\bar{\psi} \mu}^{\text {disc }} \simeq \chi_{\text {top }}$ in the left diagram of Fig. 10. Next, we show in the right panel of Fig. 10 the lattice spacing dependence of $m_{l}^{2} \chi_{\bar{\psi} \sim \mu}^{\text {disc }}$ for different temperatures, mostly above $T_{c}$ (remember that this quantity is a proxy for $\chi_{\text {top }}$ only in the symmetric phase). The required interpolations to obtain matching temperatures have used a polynomial interpolation around and slightly above the pseudocritical temperature, and the power-law fits just described for $T>300 \mathrm{MeV}$. We then consider two continuum extrapolations, $m_{l}^{2} \chi_{\bar{\psi} \psi}^{\mathrm{disc}}\left(a^{2}\right)=m_{l}^{2} \chi_{\bar{\psi} \psi}^{\mathrm{disc}}+O\left(a^{2}\right)$ and $m_{l}^{2} \chi_{\bar{\psi} \psi}^{\mathrm{disc}}\left(a^{2}\right)=$ $m_{l}^{2} \chi_{\bar{\psi} \sim \mu}^{\text {disc }}+K a^{2}+O\left(a^{4}\right)$, i.e., we truncate the $a^{2}$ series for $m_{l}^{2} \chi_{\bar{\psi} \mu}^{\text {disc }}$ at the zeroth (dashed lines) or first order (solid lines in Fig. 10). In the first case we consider the two points at the smallest lattice spacings, and we note that ensemble $\mathrm{A}$ is anyway well described within errors; in the second case we use the three points we have at our disposal. All fits fare nicely through the data, with continuum-extrapolated results for $m_{l}^{2} \chi_{\bar{\psi} \psi}^{\text {disc }}$ in good agreement with each other, and with the results at the smallest lattice spacing. A word of warning is however in order: it is obvious that a robust continuum extrapolation would require at least another lattice spacing. At the moment, within the given restrictions and with this caveat issued, we conclude that we observe good scaling and that using results on finite lattices as estimates of continuum ones is legitimate. We also note that the scaling pattern we observe is quite comparable with those reported by the ETMC at zero temperature in their study of the topological susceptibility from the twisted mass Dirac operator spectrum [45].

\section{Pion mass dependence and rescaling}

Our results have been obtained with pion masses ranging from 210 to $470 \mathrm{MeV}$, above the physical pion mass. The same dilute instanton gas model has a prediction for the mass scaling which may be used to extrapolate our results to the physical pion mass: $\chi_{\text {top }} \propto m_{\pi}^{4}$. We note that this leading mass dependence is more general than DIGA and simply follows from the analyticity of the chiral condensate in the chiral limit above $T_{c}$. In fact, taking $\langle\bar{\psi} \psi\rangle=$ $\sum_{n=0} a_{n} m_{l}^{2 n+1}$ in the symmetric phase, the total susceptibility is an even series in the quark mass

$$
\chi=\frac{V}{T} \frac{\partial}{\partial m_{l}}\langle\bar{\psi} \psi\rangle \equiv \chi_{\bar{\psi} \mu}^{\mathrm{disc}}+\chi_{\bar{\psi} \psi}^{\mathrm{conn} \psi}=\sum_{n=0} a_{n} m_{l}^{2 n}
$$

Barring unexpected cancellation we may assume that the same holds for the connected and disconnected susceptibilities separately; hence

$$
\chi_{\text {top }}=m_{l}^{2} \chi_{\bar{\psi} \psi}^{\mathrm{disc}}=\sum_{n=0} a_{n} m_{\pi}^{4(n+1)} .
$$

An exact DIGA form would imply that the leading order is exact, i.e., that the disconnected chiral susceptibility does not depend on the pion mass in the mass range considered. Our results do show a mass dependence, which is not surprising, given the relatively large masses that we are using: smaller masses are probably needed to get rid of the subleading mass corrections. In this first study, rather than attempting an extrapolation in mass we content ourselves with the simple rescaling dictated by the leading order, as was done first in Ref. [18].

In Fig. 12 we present the results for the topological susceptibility obtained by rescaling the data for different pion masses to the physical pion mass according to the leading scaling prescription. For $m_{\pi}=370 \mathrm{MeV}$, the continuum result from the first-order extrapolation in $a^{2}$ is also given, which is consistent with the $\mathrm{D}$-ensemble data points within error bars. In the same diagram we reproduce the results from Table S7 of Ref. [9] obtained with physical quark masses for comparison. As we have already discussed, the trend close to $T_{c}$ is different, while there is a broad agreement at high temperatures; we will discuss below the implications of the residual differences on the axion mass. A similar agreement holds with the results of Ref. [19].

\section{Axions}

A power-law decay for the topological susceptibility as noted by many authors opens a very interesting possibility: a safe extrapolation to very large temperatures. This feature has been exploited in applications to axion physics, as briefly mentioned in the Introduction. In a nutshell (see e.g., Ref. [46]), when the axion mass is of the order of the inverse of the Hubble parameter, the axion starts to oscillate: $3 H(T)=m_{a}(T)=\sqrt{\chi_{\text {top }}(T)} / f_{a} . f_{a}$ can be

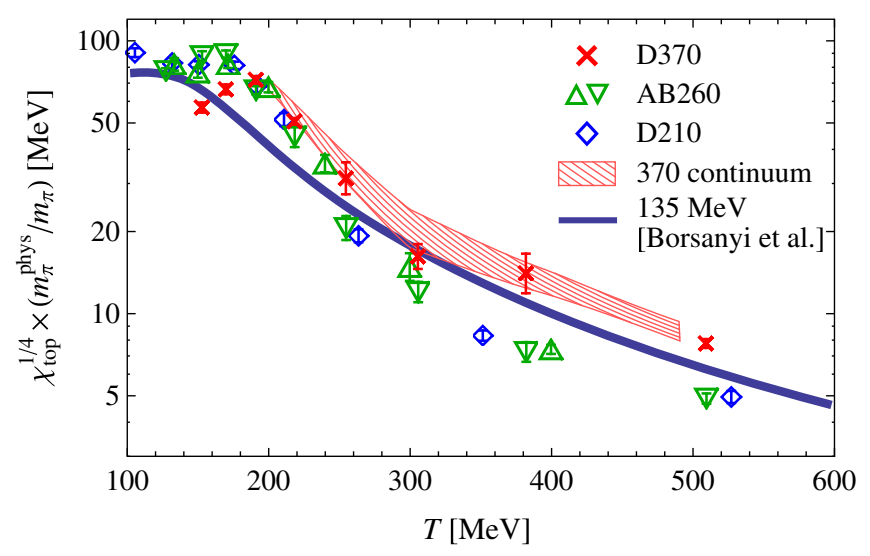

FIG. 12. The fourth root of the topological susceptibility versus the temperature for three different pion masses, rescaled to the physical pion mass according to $\chi_{\text {top }} \propto m_{\pi}^{4}$. For the $m_{\pi}=$ $370 \mathrm{MeV}$ continuum band the solid line extrapolations from the right panel of Fig. 10 are taken. We also superimpose the tabulated results (Table S7) from Ref. [9]. 
traded for the zero-temperature topological susceptibility and the axion mass via $\chi_{\text {top }}=f_{a}^{2} m_{a}^{2}$, and hence the knowledge of the temperature dependence of the topological susceptibility suffices to determine the time of the beginning of oscillation. At this time, the energy density $\rho_{a}(T)$ of the oscillating axion field is the same as a collection of axions at rest $\rho_{a}(T) \approx 1 / 2 m_{a}^{2}(T) f_{a}^{2} \theta^{2}$, and the number density $n_{a}(T)=\rho_{a}(T) / m_{a}(T)$ can be estimated as $n_{a}(T) \approx 1 / 2 m_{a}(T) f_{a}^{2} \theta^{2}$. The axion-to-entropy ratio remains constant after the beginning of the oscillations, so the present mass density of axions is $\rho_{a, 0}=\frac{n_{a}}{s} m_{a} s_{0}$, where $s, s_{0}$ are the entropies at time $T$ and today, and $\Omega_{a}=\frac{\rho_{a, 0}}{\rho_{c}}$, where $\rho_{c}$ is the critical density. To obtain simple expressions in closed form we employ the power-law parametrization $g^{*}(T)=50.8(T /(\mathrm{MeV}))^{0.053}$ for the number of relativistic d.o.f. $g^{*}(T)$ entering the Hubble parameter and entropy density, which reproduces the results up to a few percent in the temperature interval $800 \mathrm{MeV}<T<1500 \mathrm{MeV}$. Following e.g., Ref. [46] we finally arrive at $\rho_{a}\left(m_{a}\right) \propto m_{a}^{-\frac{3.053+d / 2}{2.027+d / 2}}$, where $d>0$ defines the power-law decay of the topological susceptibility at high temperatures discussed above, $\chi_{\text {top }} \simeq A T^{-d}$, and we use the latest Particle Data Group results for the required astrophysical constants [47]. We present the results graphically in Fig. 13: we plot the axion's fractional contribution to dark matter versus the axion mass for various situations. Similar analyses have been presented in previous works, and we confirm to a large extent their conclusions $[9,19,48]$. The first three lines are obtained from our results, rescaled to the physical pion mass. We have included errors for the lowest mass, which are not visible in the graph. The discrepancy between the results

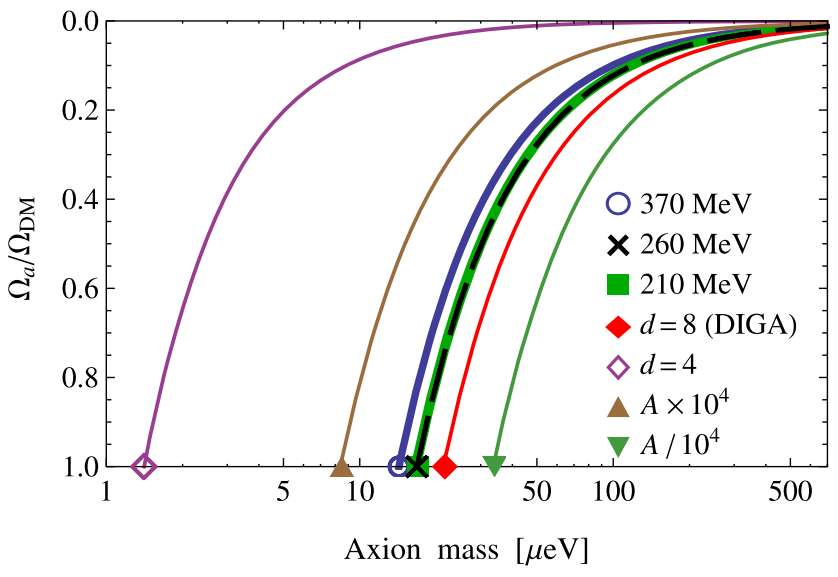

FIG. 13. The axion contribution to dark matter versus the axion mass: the first three lines show our results for three pion masses 370,260 , and $210 \mathrm{MeV}$, respectively, all rescaled to the physical pion mass according to $\chi_{\text {top }} \propto m_{\pi}^{4}$. The other lines are mock data meant to study the sensitivity of the $210 \mathrm{MeV}$ curve to the fit parameters of the topological susceptibility $\chi_{\text {top }} \simeq A T^{-d}$. for the two lowest pion masses, and for a pion of $370 \mathrm{MeV}$ may be ascribed to violations of the mass scaling discussed above, and are anyway small at a practical level. Clearly, as we know, further uncertainties might be hidden in the results: to estimate their impact we plot a few mock curves on the basis of $210 \mathrm{MeV}$ data. First, we keep the same amplitudes as the one we have measured, but choose the DIGA exponent. Second, we choose a small exponent similar to the one reported in Ref. [18]. To study the effect of the amplitude, we fix the exponent to the one we have measured at $210 \mathrm{MeV}$, and consider the fourth root of the amplitude 10 times larger or smaller. In all cases the intercept with the abscissa (overclosure bound) defines the absolute lower bound for the axion mass: for our results, this gives $m_{a} \simeq 20 \mu \mathrm{eV}$. This limit becomes more stringent if we assume that the axions only contribute to a fraction of DM;- the results can be read off of the plot from the intercept of the desired fraction and the corresponding curves. Clearly the bounds are robust against "small" changes of parameters. However a significant variability remains. In particular, as also noted in Ref. [49], slower decays (as those observed by us using the gluonic definition of the topological susceptibility) would considerably lower the axion bound.

\section{DISCUSSION}

We have studied chiral and topological properties of $N_{f}=2+1+1$ QCD with twisted mass Wilson fermions. The strange and charm mass have their physical values, while for the light pion we have considered four different masses.

We have identified the pseudocritical temperature as a function of the pion mass. We have found that an extrapolation to the pseudocritical temperature for the physical pion is robust with respect to different assumptions for the universality class of the two-flavor massless theory, and agrees well with previous estimates. This serves as a sanity check of the twisted mass chiral dynamics around $T_{c}$ and in addition it confirms the irrelevance of a dynamical charm in the transition region.

We have measured the topological susceptibility in the range $150-500 \mathrm{MeV}$ and found a rather fast decrease with temperature in the range $T_{c}<T \lesssim 350 \mathrm{MeV}$. This feature is predicted in recent instanton-dyon models [44]; however it has not been observed in other recent lattice studies. Such a fast decrease may also be understood within the framework of the QCD magnetic equation of state [50], as it is known that around $T_{c}$ the disconnected susceptibility almost saturates the total susceptibility $\partial\langle\bar{\psi} \psi\rangle / \partial m$. Since the total susceptibility in the symmetric phase behaves as $1 /\left(T-T_{c}\right)^{\gamma}$ with $\gamma \simeq 1$ (with a weak mass dependence due to Griffith analyticity) it is rather natural to expect a fast decrease of the topological susceptibility leading to an apparently large exponent in a simple power-law parametrization $T^{\alpha}$. This scenario should be checked by directly 
computing the regular contribution to the susceptibility, and most importantly the $\gamma_{5}$ susceptibility, which would allow the computation of the topological susceptibility without relying on the restoration of the chiral symmetry. At higher temperatures the contribution from the regular part becomes significant and the results approach the DIGA behavior from above; however, as we have already mentioned it is hard to exclude a continual decreasing trend which would bring the results well below that.

This work might be extended along several directions. First - and obviously — the results should be further pushed towards lower masses. The first zero-temperature results by the ETMC with $N_{f}=2+1+1$ and a physical pion mass have appeared recently [51], and we hope to be able to extend them to high temperatures. Second, we would like to clarify in detail the source of discrepancies among different methods. Although there are several reasons to believe that these discrepancies may be traced back to lattice artifacts, we are still in need of clear evidence. In a companion paper we will present results with a gluonic method obtained on the same lattices [42], and a study using the overlap operator is in progress [42]. Last but not least, we hope to go beyond the second-order cumulant studied here in order to learn more about the axion potential.

\section{ACKNOWLEDGMENTS}

We are grateful to the Supercomputing Center of Lomonosov Moscow State University, to the HybriLIT group of JINR, to the HLRN and to CINECA (INFNCINECA agreement) for computational resources. E.-M. I. and M.P. L. wish to thank the Director and the Staff at the European Center for Theoretical Nuclear Physics, Trento, for their kind support and hospitality, and the Theory Group at Justus Liebig Universität in Giessen. M. P. L. thanks the Bogoliubov Laboratory for Theoretical Physics of JINR Dubna and the Galileo Galilei Institute for Theoretical Physics for hospitality. This work is partially supported by the COST (European cooperation in Science and Technology), Action CA 15213, Theory of Hot Matter in Ultrarelativistic Heavy Ion Collisions THOR and Russian Foundation for Basic Research RFBR Grant No. 18-02-01107. A. T. acknowledges support from the Foundation for the Advancement of Theoretical Physics and Mathematics (BASIS).
[1] H. T. Ding, F. Karsch, and S. Mukherjee, Int. J. Mod. Phys. E 24, 1530007 (2015).

[2] C. Schmidt and S. Sharma, J. Phys. G 44, 104002 (2017).

[3] P. de Forcrand and M. D'Elia, Proc. Sci. LATTICE2016 (2017) 081.

[4] F. Cuteri, C. Czaban, O. Philipsen, and A. Sciarra, EPJ Web Conf. 175, 07032 (2018).

[5] S. Sharma (HotQCD Collaboration), arXiv:1801.08500.

[6] A. Tomiya, G. Cossu, S. Aoki, H. Fukaya, S. Hashimoto, T. Kaneko, and J. Noaki, Phys. Rev. D 96, 034509 (2017).

[7] M. Laine and Y. Schroder, Phys. Rev. D 73, 085009 (2006).

[8] A. Bazavov et al. (MILC Collaboration), Proc. Sci. LATTICE2013 (2014) 154.

[9] S. Borsanyi et al., Nature (London) 539, 69 (2016).

[10] F. Burger, E.-M. Ilgenfritz, M. P. Lombardo, M. MüllerPreussker, and A. Trunin, J. Phys. Conf. Ser. 668, 012092 (2016).

[11] F. Burger, G. Hotzel, M. Müller-Preussker, E.-M. Ilgenfritz, and M. P. Lombardo, Proc. Sci. LATTICE2013 (2013) 153.

[12] S. Borsanyi, Z. Fodor, C. Hoelbling, S. D. Katz, S. Krieg, C. Ratti, and K. K. Szabo (Wuppertal-Budapest Collaboration), J. High Energy Phys. 09 (2010) 073.

[13] A. Bazavov et al. (HotQCD Collaboration), Phys. Rev. D 85, 054503 (2012).

[14] T. Bhattacharya et al. (HotQCD Collaboration), Phys. Rev. Lett. 113, 082001 (2014).

[15] M. Müller-Preussker, Proc. Sci. LATTICE2014 (2015) 003.

[16] C. Bonati, EPJ Web Conf. 175, 01011 (2018).
[17] A. Trunin, F. Burger, E.-M. Ilgenfritz, M. P. Lombardo, and M. Müller-Preussker, J. Phys. Conf. Ser. 668, 012123 (2016).

[18] C. Bonati, M. D’Elia, M. Mariti, G. Martinelli, M. Mesiti, F. Negro, F. Sanfilippo, and G. Villadoro, J. High Energy Phys. 03 (2016) 155.

[19] P. Petreczky, H. P. Schadler, and S. Sharma, Phys. Lett. B 762, 498 (2016).

[20] Y. Taniguchi, K. Kanaya, H. Suzuki, and T. Umeda (WHOTQCD Collaboration), Phys. Rev. D 95, 054502 (2017).

[21] J. B. Kogut, J. F. Lagae, and D. K. Sinclair, Phys. Rev. D 58, 054504 (1998).

[22] A. Bazavov et al. (HotQCD Collaboration), Phys. Rev. D 86, 094503 (2012).

[23] M. I. Buchoff et al. (LLNL/RBC Collaboration), Phys. Rev. D 89, 054514 (2014).

[24] O. Wantz and E. P. S. Shellard, Phys. Rev. D 82, 123508 (2010).

[25] E. Berkowitz, M. I. Buchoff, and E. Rinaldi, Phys. Rev. D 92, 034507 (2015).

[26] S. Borsanyi, M. Dierigl, Z. Fodor, S. D. Katz, S. W. Mages, D. Nogradi, J. Redondo, A. Ringwald, and K. K. Szabo, Phys. Lett. B 752, 175 (2016).

[27] P. T. Jahn, G. D. Moore, and D. Robaina, Phys. Rev. D 98, 054512 (2018).

[28] F. Burger, E.-M. Ilgenfritz, M. P. Lombardo, M. MüllerPreussker, and A. Trunin, Nucl. Phys. A967, 880 (2017).

[29] R. Frezzotti and G. C. Rossi, J. High Energy Phys. 08 (2004) 007. 
[30] A. Shindler, Phys. Rep. 461, 37 (2008).

[31] C. Alexandrou, V. Drach, K. Jansen, C. Kallidonis, and G. Koutsou, Phys. Rev. D 90, 074501 (2014).

[32] T. Umeda, S. Aoki, S. Ejiri, T. Hatsuda, K. Kanaya, H. Ohno, and Y. Maezawa (WHOT-QCD Collaboration), Phys. Rev. D 85, 094508 (2012).

[33] Y. Aoki, Z. Fodor, S. D. Katz, and K. K. Szabo, Phys. Lett. B 643, 46 (2006).

[34] R. Sommer, Nucl. Phys. B411, 839 (1994).

[35] F. Burger, E.-M. Ilgenfritz, M. Kirchner, M. P. Lombardo, M. Müller-Preussker, O. Philipsen, C. Urbach, and L. Zeidlewicz (tmfT Collaboration), Phys. Rev. D 87, 074508 (2013).

[36] M. Cheng et al., Phys. Rev. D 77, 014511 (2008).

[37] K. Osterwalder and E. Seiler, Ann. Phys. (N.Y.) 110, 440 (1978).

[38] J. M. Pendlebury et al., Phys. Rev. D 92, 092003 (2015).

[39] R. D. Peccei and H. R. Quinn, Phys. Rev. Lett. 38, 1440 (1977); Phys. Rev. D 16, 1791 (1977).

[40] S. Weinberg, Phys. Rev. Lett. 40, 223 (1978).

[41] F. Wilczek, Phys. Rev. Lett. 40, 279 (1978).
[42] A. Trunin, F. Burger, E.-M. Ilgenfritz, and M. P. Lombardo (to be published).

[43] G. Aarts, S. Kim, M. P. Lombardo, M. B. Oktay, S. M. Ryan, D. K. Sinclair, and J.-I. Skullerud, Phys. Rev. Lett. 106, 061602 (2011).

[44] R. Larsen and E. Shuryak, arXiv:1703.02434.

[45] K. Cichy, E. Garcia-Ramos, and K. Jansen (ETM Collaboration), J. High Energy Phys. 02 (2014) 119.

[46] M. S. Turner, Phys. Rev. D 33, 889 (1986).

[47] C. Patrignani et al. (Particle Data Group), Chin. Phys. C 40, 100001 (2016); M. Tanabashi et al. (Particle Data Group), Phys. Rev. D 98, 030001 (2018).

[48] V. B. Klaer and G. D. Moore, J. Cosmol. Astropart. Phys. 11 (2017) 049.

[49] G. Grilli di Cortona, E. Hardy, J. Pardo Vega, and G. Villadoro, J. High Energy Phys. 01 (2016) 034.

[50] S. Ejiri, F. Karsch, E. Laermann, C. Miao, S. Mukherjee, P. Petreczky, C. Schmidt, W. Soeldner, and W. Unger, Phys. Rev. D 80, 094505 (2009).

[51] J. Finkenrath et al., EPJ Web Conf. 175, 02003 (2018). 